\title{
A compact echelle spectrograph for characterization of astro-combs
}

\author{
Rafael A. Probst ${ }^{1} \cdot$ Tilo Steinmetz $^{2}$ - Yuanjie Wu ${ }^{1,2} \cdot$ Frank Grupp $^{3} \cdot$ Thomas Udem $^{1}$ • \\ Ronald Holzwarth ${ }^{1,2}$
}

Received: 1 September 2016 / Accepted: 26 December 2016 / Published online: 28 February 2017

(c) The Author(s) 2017. This article is published with open access at Springerlink.com

\begin{abstract}
We present an echelle spectrograph that is optimized for characterization of frequency combs for astronomical applications (astro-combs). In spite of its very compact and cost-efficient design, it allows viewing the spectrum of a frequency comb in nearly the same way as a full-sized high-resolution echelle spectrograph as used at astronomical observatories. This is of great value for testing and characterizing astro-combs during their assembly phase. The spectrograph can further be utilized to effectfully demonstrate the remarkable capabilities of astro-combs.
\end{abstract}

\section{Introduction}

At the beginning of this millennium, the advent of laser frequency combs (LFCs) [1] has paved the way to pushing precision spectroscopy to a new extreme. Through this invention, the uncertainty of $1 \mathrm{~S}-2 \mathrm{~S}$ transition

This article is part of the topical collection "Enlightening the World with the Laser" - Honoring T. W. Hänsch guest edited by Tilman Esslinger, Nathalie Picqué, and Thomas Udem.

Electronic supplementary material The online version of this article (doi:10.1007/s00340-016-6628-0) contains supplementary material, which is available to authorized users.

Rafael A. Probst

rprobst@mpq.mpg.de

1 Max-Planck-Institut für Quantenoptik, Hans-Kopfermann-Str. 1, 86748 Garching, Germany

2 Menlo Systems GmbH, Am Klopferspitz 19a, 82152 Martinsried, Germany

3 Universitäts-Sternwarte München, Scheinerstr. 1, 81679 München, Germany measurements in atomic hydrogen was brought down to the $10^{-15}$ level, for a precision test of quantum electrodynamics [2]. It was largely for this achievement that the pioneers of laser spectroscopy T. W. Hänsch and J. L. Hall were awarded the Nobel Prize of Physics in 2005.

As the LFC quickly found manifold applications, it was recognized in 2007 that also astronomy would greatly profit from a vast leap in precision enabled by the LFC [3]. An LFC provides a multitude of sharp, evenly spaced spectral lines (modes), whose optical frequencies can be phasecoherently linked to an atomic clock. The frequency of the $n$th mode is $f_{n}=n \times f_{r}+f_{0}$, with the mode spacing $f_{r}$ and the offset frequency $f_{0}$. For astronomy, this provides a nearly ideal calibrator for high-resolution echelle spectrographs. Conventional calibration sources, such as thoriumargon arc lamps, presently limit the precision of astronomical spectrographs through their irregular line pattern and line drifts with aging of the lamp.

LFCs optimized for astronomical applications (astrocombs) have thus been developed [4-6], which promise discoveries of Earth-like exoplanets through radialvelocity measurements and a more precise constraint on a potential cosmic variability of fundamental constants [7]. A direct measurement of the acceleration of the cosmic expansion also appears within reach [8]. Several important spectrographs at major observatories, such as HARPS [9, 10], HARPS-N [4], VTT [11] and FOCES [12, 13], have recently been equipped with astro-combs, and others, such as ESPRESSO [14], are about to follow.

For spectrograph calibration tests, several astro-combs have temporarily been deployed at astronomical observatories. Such campaigns represent a major effort, as astro-combs are complicated and sensitive systems to be transported to remote places, and measurement time at observatories is in high demand. Some aspects of 
astro-combs can easily and accurately be characterized without the need for an echelle spectrograph: the mode spacing $f_{r}$ is exactly the repetition rate of the emitted laser pulses detectable with a photodiode. The spectral coverage can be measured with a small low-resolution spectrometer, which is already integrated in many astro-combs [15]. For the characterization of a few other aspects, however, there is hardly an alternative to the use of an echelle spectrograph: studying line-by-line intensity fluctuations or a possible continuum background between the lines requires resolving individual lines over the full spectral width. Yet, the mode spacing is adapted to a high-resolution echelle spectrograph, and the selection of lab instruments with a similar resolution and bandwidth is very limited. Investigations of these properties are however highly relevant for applications [10].

It would thus be convenient to have a spectrograph directly in place, where astro-combs are being built, to characterize them before shipping them to their destinations. The state-of-the-art astronomical spectrographs are, however, elaborate machines of respectable cost and size. Yet, as shown in this article, a spectrograph explicitly built for astro-comb characterization can be compact and inexpensive.

Theodor W. Hänsch, to whom this journal issue is dedicated on the occasion of his 75th birthday, has always sought to find simple yet effective solutions. This spirit has led him to inventing phase-stabilized LFCs. Sometimes, his experiments playfully involve everyday items, such as jelly as an active laser material [16] or a toy railroad for motion control of optical elements [17], while at the same time yielding important scientific results. Following this school of thought, we opt for elegantly simple solutions for our spectrograph. We show how off-the-shelf laboratory components and photographic equipment can be used to create a valuable measurement tool.

\section{Spectrograph design}

\subsection{A note on spectrograph size}

What allows us to drastically shrink down our spectrograph is the possibility of using a single-mode optical fiber as a spectrograph input. Being a fiber-laser system, the output of the astro-comb is intrinsically single mode. This is different for stars, which are blurred into a non-diffraction-limited disk by atmospheric seeing. For applications other than solar astronomy, where optical power is abundant, this light is thus commonly fed into multimode fibers to preserve efficient coupling to the spectrograph. The fiber exit is the spectrograph entrance slit, whose multimode nature precludes the spectrograph from reaching a diffraction-limited resolution. A typical astronomical spectrograph hence needs to be much bigger than ours, to illuminate a larger number of grating grooves, to ultimately reach the same resolution.

Since bigger telescopes capture more seeing elements, and thus greater numbers of spatial modes, the size of the spectrograph scales with that of the telescope. The resulting trend towards bigger spectrographs may soon be stopped by continuing progress in adaptive optics [18]. Several single-mode spectrographs are currently under development for red to near-infrared wavelengths [19-21], where "extreme" adaptive optics systems are starting to allow single-mode fiber injection with reasonable efficiency [22].

As an input fiber for our spectrograph, we use a single-mode fiber (Thorlabs S405-XP) designed for a wavelength range of 400-680 $\mathrm{nm}$. The fiber core has a modefield diameter of $4 \mu \mathrm{m}$ and a numerical aperture (NA) of 0.12. For comparison, HARPS uses a $70 \mu \mathrm{m}$ fiber core, which is collimated into a beam of $21 \mathrm{~cm}$ in diameter. Assuming a similar fiber NA and echelle grating, our 20 times smaller fiber core means that we can reach a similar resolution as HARPS with a beam of just $1 \mathrm{~cm}$. We can thus conveniently build our spectrograph from standard 1 inch optics.

\subsection{Optical setup}

We construct our spectrograph following the optical layout shown in Fig. 1. The fiber output within the spectrograph is polished into a ceramic ferrule (without connector), which is held in place by a small clamp mount, and connected to the fiber jacket with a heat shrink tube. The fiber output is collimated into a Gaussian beam of $9.5 \mathrm{~mm}$ full-width at half-maximum (FWHM). The collimator

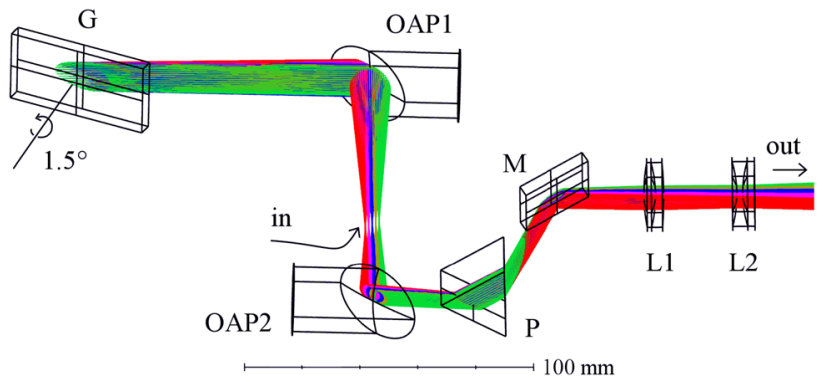

Fig. 1 Spectrograph optical layout. Rays in different colors represent different wavelengths within the same diffraction order. In Fiber input, $O A P 1 / O A P 290^{\circ}$ off-axis parabolic mirror with $101.6 / 50.8 \mathrm{~mm}$ effective focal length, $G$ echelle grating, turned by $1.5^{\circ}$ around its surface normal, $P$ N-SF11 equilateral prism, $M$ plane mirror, $L 1 / L 2$ lens telescope consisting of two achromatic doublets with 125/-100 mm focal length 
is a $90^{\circ}$ off-axis parabolic mirror (OAP1 in Fig. 1) with a $101.6 \mathrm{~mm}$ effective focal length (EFL). The $90^{\circ}$ angle was chosen, because it is the most common in optics catalogs, so mirrors with suitable focal lengths were available off-the-shelf with good optical quality (surface accuracy $\lambda / 8$ at $630 \mathrm{~nm}$, roughness $<50 \AA$ root-mean-square, supplier: Edmund Optics).

Ten centimeters after OAP1, we place a $50 \times 25 \mathrm{~mm}^{2}$ echelle grating with 31.6 grooves $/ \mathrm{mm}$ and a $63^{\circ}$ blaze angle (Thorlabs GE2550-0363). The grating is positioned, such that at the center of each diffraction order, the beam is precisely reflected back to OAP1 (Littrow configuration). From this initial position, the grating is turned by $1.5^{\circ}$ around its surface normal. This creates an upward displacement of the beam of $2.6 \mathrm{~mm}$ after OAP1, allowing it to pass above the input fiber without getting obstructed. A dispersed intermediate focus is formed above the fiber end, which can be utilized to intercept stray light from the grating by inserting a slit.

After the intermediate focus, the light is collimated by another $90^{\circ}$ off-axis parabolic mirror (OAP2), with an EFL of $50.8 \mathrm{~mm}$. The shorter EFL of OAP2 demagnifies the beam diameter to $4.7 \mathrm{~mm}$ FWHM, which facilitates the handling of the dispersed beam with 1 in wide optical components without clipping it. Furthermore, it steepens the dispersion angle, which magnifies the echellogram image in horizontal direction. This can be understood as being a direct consequence of the reduced beam diameter, as the magnification of the image is required to preserve the Lagrange invariant. In our optical setup, this effect allows us to obtain an image of equal size with a shorter focal length of the subsequent lens system.

As an echelle grating produces numerous diffraction orders, a cross disperser is an essential part of any echelle spectrograph. Its dispersion is perpendicular to that of the grating to separate the echelle orders from one another. For this, we employ an equilateral prism that we place after OAP2. As this is after the location where the angular dispersion is doubled, we need a relatively strong cross-dispersion. We thus choose N-SF11 as a prism material, for its high refractive index and low Abbe number. This choice comes with the minor disadvantage of a relatively unequal angular separation of the orders, with the blue ones being separated more widely than the red ones. The strongly dispersive prism also causes the echelle orders to have a pronounced curvature. Light on the side of an echelle order passes through the prism in a different angle, and is, therefore, deflected differently.

As the beam is deflected upwards by the prism, we insert a fold mirror to get it back into the horizontal plane. This is followed by a sequence of two achromatic doublet lenses with 125 and $-100 \mathrm{~mm}$ focal length, respectively, mounted in an expandable tube. The total focal length of

the lens system is controlled by the relative distance of the two lenses. With this, we can project the echellogram on a laboratory wall. At a distance of $3 \mathrm{~m}$, this yields a roughly $30 \times 20 \mathrm{~cm}^{2}$ large image, creating an impressive and enjoyable visual demonstration for lab visitors. For measurements, we replace the lens telescope by a commercial camera system (see Sect. 3).

\subsection{Aberrations}

The $4.7 \mathrm{~mm}$ FWHM Gaussian beam focused over a $3 \mathrm{~m}$ distance should ideally yield a spot of $154 \mu \mathrm{m}$ FWHM at a wavelength of $550 \mathrm{~nm}$. For an astro-comb with an $18 \mathrm{GHz}$ mode spacing, the spot separation becomes $778 \mu \mathrm{m}$, which can be resolved with the naked eye. A focal length of at least $3 \mathrm{~m}$ is thus appropriate for the purpose of a visual demonstration. The visibility can be further enhanced by holding a piece of paper at an angle into the image to stretch it. The spectral resolution $R=\lambda / \delta \lambda$ should theoretically amount to 154,000 . It depends on aberrations in our optical system, whether this resolution is in fact reached. Therefore, we analyze the optical layout of Fig. 1 with Zemax. The simulated rays cover the beam FWHM. We compare the resulting spots to the FWHM as calculated from the theory of Gaussian beams (see Fig. 2). Usually, illumination of the full aperture is simulated and compared to the airy disk, but in our case, the Gaussian FWHM provides a clearer picture of the expected spectral resolution.

The main source of aberrations in our system is the fact that the beam bundles propagate off the optical axis between OAP2 and OAP1. Aberrations thus arise on both OAPs, which would add up if the OAPs were oriented parallel to one another (resulting in a U-shaped beam path). In our configuration, where the OAPs are antiparallel, aberrations from OAP1 are largely canceled by those from OAP2. For this compensation to work, it is irrelevant whether or not OAP1 and OAP2 have the same EFL. The residual aberrations are least pronounced

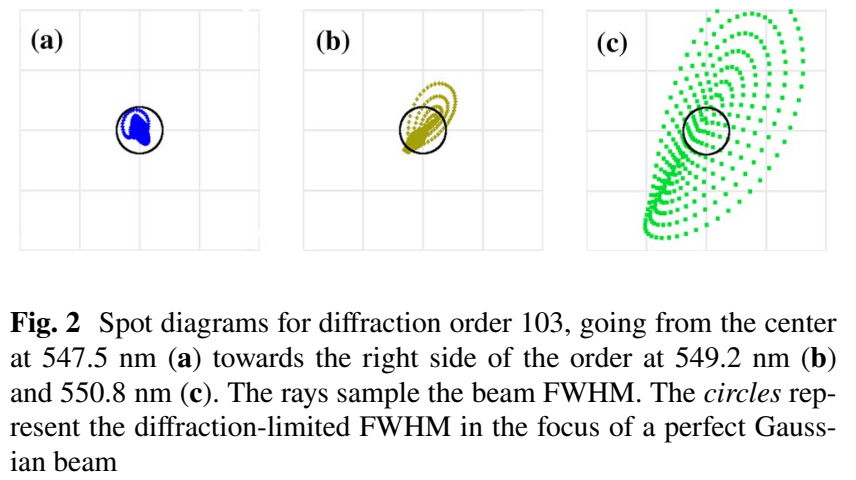


for beams at the center of the diffraction orders (Fig. 2a) as they are only displaced vertically from the optical axis by $2.6 \mathrm{~mm}$. The simulated focal spot is a tilted ellipse, which is well within the diffraction limit. In addition, we see a structure extending upwards from the ellipse caused by spherical aberration of the lens system. Beams that additionally suffer from a lateral displacement of $1.2 \mathrm{~mm}$ (Fig. 2b) start being non-diffraction-limited, and after another $1.2 \mathrm{~mm}$ (Fig. 2c, outer wing of echelle order) the comb lines start blending.

The main difference between the lateral and vertical beam displacement is that the vertical displacement is the same for all beams, so the lens system can be adjusted for the resulting defocusing. The lateral displacement changes across the echelle order, which causes the image plane to be curved. In many spectrographs, this effect is corrected by the camera optics [20]. The effect is also smaller in most spectrographs, because of the use of OAPs with smaller angles, which diminishes the aberrations. Our simulations show that with a $30^{\circ} \mathrm{OAP}$ angle, we could reach a $20-30 \%$ smaller spot size in most places where the spots are nondiffraction limited. It appears reasonable for us to trade this relatively minor improvement in for the easier availability of $90^{\circ}$ OAPs with the desired focal lengths and optical quality. In an early attempt to find the simplest acceptable solution, spherical mirrors were also tested, whose spherical aberration and coma would, however, decrease the spectrograph's resolution by a factor of 20 .

\section{Camera integration}

With a science-grade camera, the most costly part of our setup by far would be the image sensor. Astronomical cameras use CCDs with very high quantum efficiency. The CCDs are cooled, often down to $-90^{\circ} \mathrm{C}$ or even $-120^{\circ} \mathrm{C}$ to suppress dark current. This has to be done in a dry atmosphere to suppress condensation of water. However, for the sole purpose of characterizing an astro-comb, high quantum efficiency is unnecessary, since the laser system delivers vast amounts of light. Detector cooling is useful for very faint sources requiring long exposures, as the number of dark counts scales with exposure time. With the optical power available from the astro-comb, we can work with even extremely short exposures in the $\mu$ s range.

For these reasons, we want to explore the use of a consumer-grade photographic camera in our spectrograph. We use a Canon EOS M3, featuring a CMOS image sensor with $6000 \times 4000 \mathrm{px}^{2}$ and an active area of $22.3 \times 14.9 \mathrm{~mm}^{2}$ (APS-C format, $3.72 \mu \mathrm{m}$ pixel pitch). Each color channel has 14 bit of depth, which is preserved by recording the images in raw format (.CR2) and later converting them to FITS. The battery is replaced by a line adapter provided by

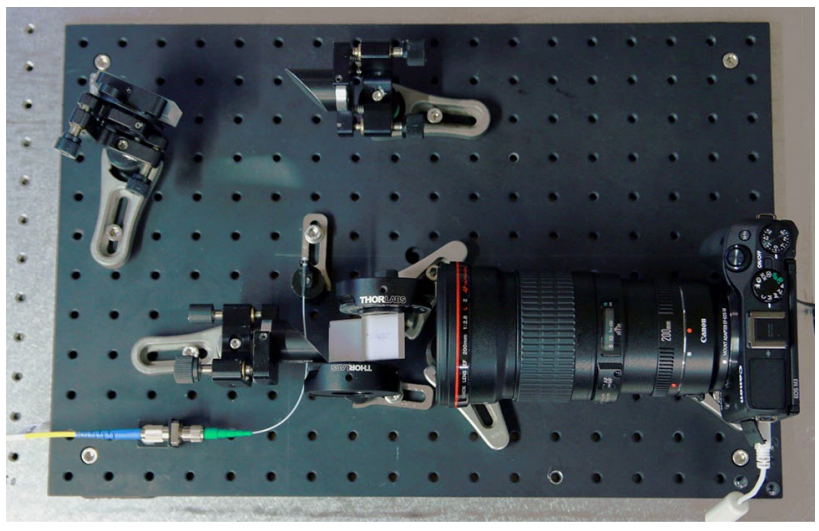

Fig. 3 Photo of the spectrograph, including camera. The physical dimensions of the black base plate are $30 \times 45 \mathrm{~cm}^{2}$

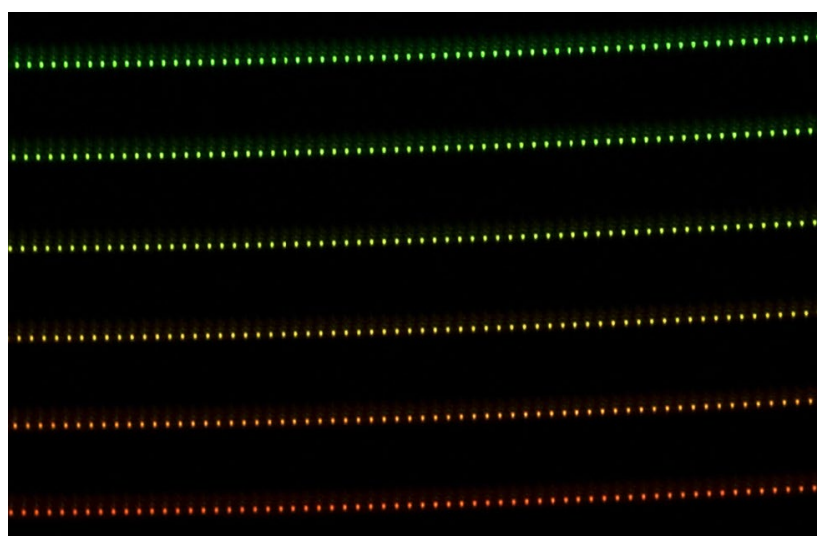

Fig. 4 Part of the echellogram near the center of the image sensor

Canon, and images are transferred via USB. Exposures are triggered remotely via WiFi or remote control. Many models also offer the possibility to both control and read out the camera via USB ("tethered shooting"), which would be simpler.

As a camera lens, we mount a prime lens with a $200 \mathrm{~mm}$ focal length (Canon EF 200mm) on the camera, which replaces the lens telescope of our setup. The complete setup, including the camera system, proves to be compact enough to be accommodated on a $30 \times 45 \mathrm{~cm}^{2}$ base plate, as shown Fig. 3. The $200 \mathrm{~mm}$ focal length of the camera lens roughly matches the size of the echellogram image to that of the image sensor. The lens aperture is set to its maximum of $7 \mathrm{~cm}$ (f/2.8), which is more than enough to avoid clipping of the beam. With this configuration, we can image 33 echelle orders onto the detector, spanning a spectral range of 477-658 $\mathrm{nm}$ (diffraction order 87-120). At around $550 \mathrm{~nm}$, a single echelle order spans $7 \mathrm{~nm}$ on the detector yielding a $32 \%$ spectral overlap from one order to the next. 
For our measurements, we use the shortest available exposure time of $250 \mu \mathrm{s}$ and set the detector sensitivity to ISO 100 to minimize noise. For the measurements presented here, we use an astro-comb with an $18 \mathrm{GHz}$ mode spacing as a light source. This astro-comb is presently in preparation for its duty on ESPRESSO. An echellogram recorded with it can be seen in Fig. 4, zoomed-in towards the center of the image to make individual comb lines visible. The full image is included as a JPEG in the supplementary material to this article. Analysis of the full frame confirms the result of the simulation that the image plane is curved cylindrically: in all echelle orders, the spots are well focused and nearly diffraction limited at the center, while moving out of focus towards the outer wings of the orders.

\section{Results}

To extract data from the recorded images, we have implemented an automatic data pipeline in Python. The pipeline first subtracts a dark image, and then sums up the three color channels, by imposing a $2 \times 2$ binning on the pixels arranged in a Bayer pattern. This yields a $3000 \times 2000 \mathrm{px}^{2}$ gray-scale image. As expected from the spot diagram (Fig. 2), the image is strongly defocused at the borders. Therefore, we crop the image to a $2000 \times 2000 \mathrm{px}^{2}$ region of interest with reasonable resolution. An echelle order at $550 \mathrm{~nm}$ spans $4.7 \mathrm{~nm}$ over this range, with $0.6 \mathrm{~nm}$ wide gaps between the orders, where spectral information is discarded. Next, the positions of the echelle orders are recognized, and each echelle order is summed up vertically over 5 (binned) pixels into a 1D data array.

The resulting 1D spectrum can be seen in Fig. 5a for order 102, centered at $553 \mathrm{~nm}$. The instrumental line shape is very well approximated by a Gaussian function, as seen from the Gaussian line fit in Fig. 5b. This is because the single-mode fiber provides a very clean Gaussian beam profile. In the center of the echelle order, the line FWHM is $1.81 \mathrm{px}$, while the line spacing is $7.44 \mathrm{px}$ (binned). This translates into a spectral resolution of 124,000 , which is about the same as that of HARPS and ESPRESSO in the standard mode. At the borders of the extracted region of the echelle order, the spectrograph resolution declines to about 65,000 due to the increasing aberrations, causing the spectral lines to slightly overlap. The maximum in resolution is somewhat lower than the 154,000 calculated above, which likely results from the fact that the Gaussian beam is truncated by the 1 in optics after 2.7 times its FWHM. Another reason might be the low-pass filter on the image sensor. This is usually a plate consisting of cemented, birefringent layers of quartz in different orientations. Its purpose to blur out the image over the $2 \times 2 \mathrm{px}^{2}$ large Bayer elements to avoid Moiré effects.
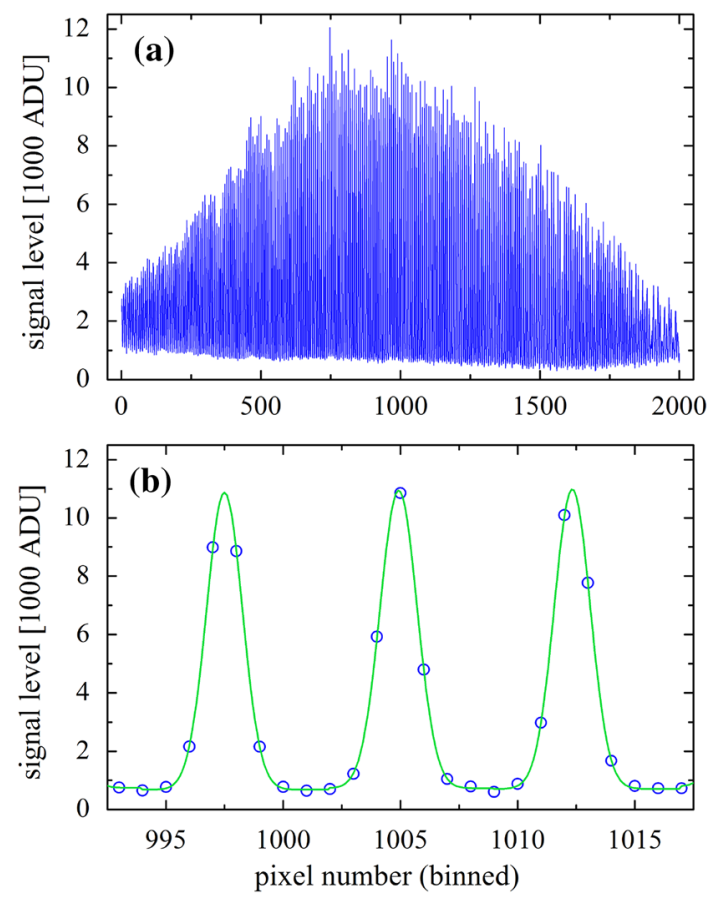

Fig. 5 Diffraction order 102 (centered at $553 \mathrm{~nm}$ ) after data extraction. a Measured data (blue line) over the full region of interest of $2000 \mathrm{px}$, after binning over $2 \times 2 \mathrm{px}^{2}$ in the Bayer matrix. b Smaller region at the center of the echelle order, with Gaussian functions (green line) fitted to the data points (blue circles). $A D U$ analog-todigital unit

As an application, we examine the spectral background of the astro-comb. It was seen in [10] that some spectral broadening schemes create an unexpectedly strong continuum background in addition to the comb lines. If an excessively strong background is not properly taken into account by the data processing pipeline of an astronomical spectrograph, it can have a severe impact on its calibration [10]. It would, therefore, be desirable to test an astro-comb for such properties before delivery to the observatory. This is, however, difficult without an echelle spectrograph because of its very particular resolution. The background can only clearly be seen when the comb lines are fully resolved without any residual overlap. An excessively high resolution, on the other hand, also makes it hard to see the background when the dynamic range is limited. This is because with higher resolution, the comb lines grow taller relative to the background, as their photon flux becomes more concentrated. This difficulty in measuring the background has led us to the conclusion that a laboratory echelle spectrograph was needed. The background characterization was hence our primary objective for this project. We demonstrate this as a first but nonetheless important application.

Our spectrograph allows viewing the spectrum with the same resolution as HARPS or ESPRESSO in the middle of 
the echelle orders, thus yielding the same relation between background level and comb line amplitudes. In the center of order 102 (Fig. 5b), the background is at 6\% of the amplitudes of the comb lines, as determined by the fit. The background is expected to be most intense at the center of an echelle order and to decrease along with the line intensities towards the borders (see [10]). In our spectrograph, we see a different behavior (Fig. 5a), which is supposedly the result of the changing resolution across the echelle order. In the outer wings of the order, the comb lines start to overlap, which masks the background.

For an accurate assessment of the background, it must be questioned to what extent the measured background level might be affected by a possible stray-light contribution, arising e.g. from imperfections of the echelle grating or mirror surfaces. It can easily be seen that the acquired images globally contain very little stray light: between the echelle orders, the dark-corrected signal level is more than $25 \mathrm{~dB}$ below the peak values of the comb lines. Furthermore, the spectral shaping reported in the next section attenuates the signal within an echelle order with a contrast of at least $20 \mathrm{~dB}$. However, each comb line might be surrounded by a small stray-light halo. We have investigated this question on a single isolated line by feeding the spectrograph with a frequency-doubled Nd:YAG laser at $532 \mathrm{~nm}$. Fitted with an offset-free Gaussian function, the data points follow the fit down to below $-20 \mathrm{~dB}$ from its peak. A possible stray-light contribution must, therefore, be limited to $<1 \%$, confirming that the background level measured above must be dominated by an incoherent spectral component.

The spectral background can systematically distort the calibration results, depending on its structure and how it is modeled in the data analysis. A recent study on HARPS used an astro-comb with a background level very similar to ours [10]. By fitting the slope of the background, the related systematic effect could be canceled at the $\mathrm{cm} / \mathrm{s}$ level. The background also comes with an increased statistical uncertainty, because it adds photon noise, but does not contribute to the signal of the comb lines, degrading their signal-to-noise ratio. In addition, less of the dynamic range is available for the comb lines. We calculate that a $6 \%$ background increases the photon noise by $21 \%$ as compared to the background-free case. For HARPS, this would imply an increase of the photon noise from typically $2.8 \mathrm{~cm} / \mathrm{s}$ in a single exposure to $3.4 \mathrm{~cm} / \mathrm{s}$.

\section{Happy birthday!}

Many astro-combs employ a spatial light modulator (SLM) as a programmable spectral filter [23, 15, 24]. This allows generating a comb spectrum with a flat-top shape, such that all lines can simultaneously reach the optimum signal level on the image sensor. The spectral sensitivity of a given spectrograph can be factored in by the flattening algorithm, which we might characterize for our spectrograph. We could then use our spectrograph to judge the attainable spectral flatness. Being able to resolve all spectral lines individually and simultaneously, the spectrograph can take into account line-by-line intensity variations. Such fine spectral structures lie below the resolution of the SLM. It is hence very important to assess how far they limit the attainable flatness. This has been done with HARPS [10], and can readily be done with our spectrograph.

However, since we dedicate this article to Ted Hänsch in honor of his $75^{\text {th }}$ birthday, measuring a flat spectrum appears somewhat too uninteresting to us. With the SLM being able to create very general spectral shapes with a $0.4 \mathrm{~nm}$ resolution and a $27 \mathrm{~dB}$ dynamic range, we would rather use it to modulate the spectrum so as to make a 75 appear in the echellogram. This idea has been inspired by a work published for Ted Hänsch's $60^{\text {th }}$ birthday, where the motion of a Bose-Einstein condensate was steered into writing a 60 [25]. What better way is there to congratulate the inventor of the self-referenced LFC than using an LFC?

For this experiment to work, we must calibrate the wavelength scale of our spectrograph with the spectral flattening unit. The wavelength scale of this unit might be slightly inaccurate, which is canceled if the spectrograph data use the same inaccurate scale. We do this using the SLM to create dips in the spectrum and see where these dips show up in the echellogram, to obtain the center wavelengths of the echelle orders. The wavelength scale within the echelle orders is derived from the Gaussian fit to the comb lines. With this calibration, we compute

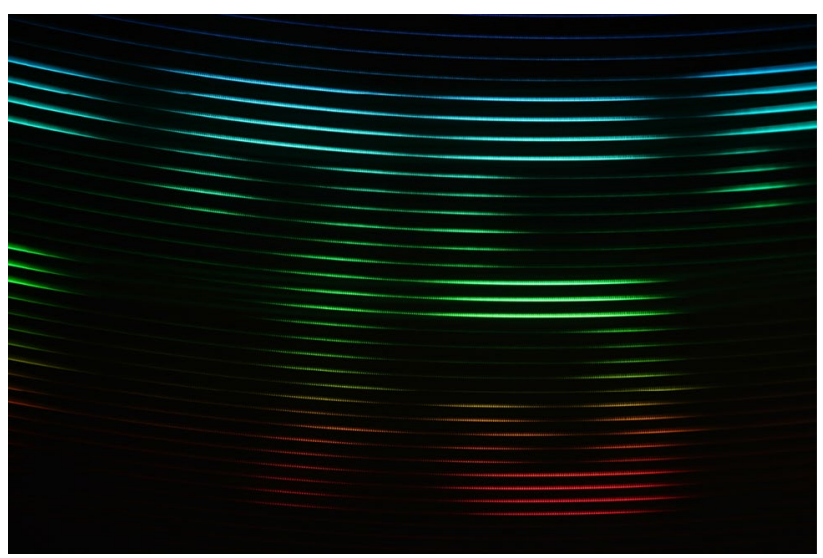

Fig. 675 , written into the echellogram of an astro-comb by reshaping its spectrum with a spatial light modulator. Happy birthday Ted Hänsch! 
a look-up table that specifies a fake spectrograph sensitivity to the flattening software. For places that we want to light up, we specify a very low sensitivity at the corresponding wavelengths, and in places that we want to remain dark, we specify a very high sensitivity.

Figure 6 shows the result. The straightness of the vertical lines is in most places nearly limited by the pixelation of the SLM that allows placing transmission windows in discrete steps only. Horizontal lines are brighter than vertical lines, because narrow spectral transmission windows inflict diffraction losses on the beam. This could be adjusted for with a further refinement of the SLM pattern. Left and right of the 75 , we see how the pattern repeats into the next order, shifted up or down, respectively, by one echelle order. The image has been overexposed to make the 75 more clearly visible. A full-resolution JPEG version of this image is included in the supplementary material.

\section{Possible upgrades and future applications}

Accuracy and long-term stability are essential features of any astro-comb, and represent the main advantage over alternative calibration methods. A stringent test of these crucial properties generally requires a comparison of two independent astro-combs on a two-channel spectrograph, as it was done during a recent campaign on HARPS [10]. Such expensive and time-consuming campaigns might become unnecessary with a high-resolution echelle spectrograph for laboratory use. Directly after assembly, each astro-comb could be compared to a reference astro-comb, to ensure that it fulfills its specifications. A laboratorybased spectrograph may also facilitate stability tests on very long time scales up to years, as needed for searching long-period exoplanets and other applications.

Making our spectrograph suitable for these important and ambitious goals requires a number of upgrades, such as extending it to a two-channel spectrograph. The large separation of the echelle orders leaves more than enough space in between them to accommodate additional orders from a second input fiber. This could be implemented using a ferrule at the spectrograph input with a hole large enough to comprise two fibers. The distance of the echelle orders from the two fibers could be controlled by turning the ferrule around its axis. For characterizing the stability down to $1 \mathrm{~cm} / \mathrm{s}$ as commonly desired in the search for Earth-like exoplanets, pressure and temperature stabilization of the echelle spectrograph are likely to be a requirement: A change in ambient air pressure of $1 \mathrm{hPa}$ alters the wavelengths equivalent to a Doppler shift of $82.5 \mathrm{~m} / \mathrm{s}$. This change might be too large to track the relative drift of two astro-combs down to $1 \mathrm{~cm} / \mathrm{s}$. Fortunately, both pressure and temperature stabilization are greatly facilitated by the small footprint of our spectrograph.

Similar to the stability, a set of two independent astrocombs can be tested for their consistency. For this purpose, two astro-combs are used for a simultaneous calibration of a two-channel spectrograph showing how well they agree on an absolute basis [10]. For an absolute calibration of a spectrograph, the comb modes need to be identified. The coarse calibration with the flattening setup used in this paper was not accurate enough for this. To identify the modes in one order, a single-frequency laser can be used, whose wavelength is measured with a wavemeter. The obtained calibration can be scaled to other orders using $\lambda_{2}=\lambda_{1} m_{1} / m_{2}$, which relates the wavelengths $\lambda_{1}$ and $\lambda_{2}$ at the same position for two different orders $m_{1}$ and $m_{2}$. To account for any possible lateral displacement of the orders relative to one another, the prism dispersion direction can be precisely measured on the image sensor by replacing the echelle grating with a plane mirror.

\section{Conclusion}

In this article, we have shown how to build a high-resolution echelle spectrograph in a simple, compact and cost-efficient way. In the center of each echelle order, our spectrograph reaches the same resolution as state-of-theart astronomical echelle spectrographs used for exoplanet hunting. As an application, we have shown how the spectrograph can be used to characterize temporally incoherent components within the astro-comb spectrum. This aspect is highly relevant for calibrating astronomical spectrographs. Its characterization was the major motivation for the development of the spectrograph we have described, next to the characterization of line-by-line intensity fluctuations. We have also discussed several upgrades that may open up the door to more advanced applications, such as the relative characterization of two astro-combs in terms of stability and consistency. The requirements for this include adding a second input fiber, as well as temperature and pressure stabilization of the spectrograph.

Acknowledgements Open access funding provided by Max Planck Society. We gratefully acknowledge Florian Kerber, Gaspare Lo Curto, Gerardo Avila, Luca Pasquini, and Antonio Manescau from the European Southern Observatory, and Hanna Kellermann from the University Observatory Munich, for collaborating with us on astrocombs, and for helpful discussions on spectrograph design.

Open Access This article is distributed under the terms of the Creative Commons Attribution 4.0 International License (http:// creativecommons.org/licenses/by/4.0/), which permits unrestricted use, distribution, and reproduction in any medium, provided you give appropriate credit to the original author(s) and the source, provide a 
link to the Creative Commons license, and indicate if changes were made.

\section{References}

1. Th. Udem, R. Holzwarth, T.W. Hänsch, Nature 416, 233-237 (2002)

2. C.G. Parthey et al., Phys. Rev. Lett. 107, 203001 (2011)

3. M.T. Murphy, Th. Udem, R. Holzwarth, A. Sizmann, L. Pasquini, C. Araujo-Hauck, H. Dekker, S. D’Odorico, M. Fischer, T.W. Hänsch, A. Manescau, Mon. Not. R. Astron. Soc. 380, 839-847 (2007)

4. A.G. Glenday, C.-H. Li, N. Langellier, G. Chang, L.-J. Chen, G. Furesz, A.A. Zibrov, F. Kärtner, D.F. Phillips, D. Sasselov, A. Szentgyorgyi, R.L. Walsworth, Optica 2, 250-254 (2015)

5. G.G. Ycas, F. Quinlan, S.A. Diddams, S. Osterman, S. Mahadevan, S. Redman, R. Terrien, L. Ramsey, C.F. Bender, B. Botzer, S. Sigurdsson, Opt. Express 20, 6631-6643 (2012)

6. T. Wilken, G. Lo Curto, R.A. Probst, T. Steinmetz, A. Manescau, L. Pasquini, J.I. González Hernández, R. Rebolo, T.W. Hänsch, Th. Udem, R. Holzwarth, Nature 485, 611-614 (2012)

7. J.K. Webb, J.A. King, M.T. Murphy, V.V. Flambaum, R.F. Carswell, M.B. Bainbridge, Phys. Rev. Lett. 107, 191101 (2011)

8. J. Liske et al., Mon. Not. R. Astron. Soc. 386, 1192-1218 (2008)

9. M. Mayor et al., The Messenger 114, 20-24 (2003)

10. R.A. Probst et al., Proc. SPIE 9908, 990864 (2016)
11. R.A. Probst, L. Wang, H.-P. Doerr, T. Steinmetz, T.J. Kentischer, G. Zhao, T.W. Hänsch, Th. Udem, R. Holzwarth, W. Schmidt, New J. Phys. 17, 023048 (2015)

12. M.J. Pfeiffer, C. Frank, D. Baumüller, K. Fuhrmann, T. Gehren, Astron. Astrophys. Suppl. Ser. 130, 381-393 (1998)

13. A. Brucalassi, F. Grupp, H. Kellermann, L. Wang, F. LangBardl, N. Baisert, S.M. Hu, U. Hopp, R. Bender, Proc. SPIE 9908, 99085W (2016)

14. D. Mégevand et al., Proc. SPIE 8446, 84461R (2012)

15. R.A. Probst et al., Proc. SPIE 9147, 91471C (2014)

16. T.W. Hänsch, Optics Photon. News 16, 14-16 (2005)

17. H.-R. Xia, S.V. Benson, T.W. Hänsch, Laser Focus 17, 54-58 (1981)

18. J. Ge, J.R.P. Angel, D.G. Sandler, J.C. Shelton, D.W. McCarthy, J.H. Burge, Proc. SPIE 3126, 343-354 (1997)

19. J.R. Crepp et al., Proc. SPIE 9908, 990819 (2016)

20. C. Schwab et al., Proc. SPIE 9912, 991274 (2016)

21. A.D. Rains, M.J. Ireland, N. Jovanovic, T. Feger, J. Bento, C. Schwab, D.W. Coutts, O. Guyon, A. Arriola, S. Gross, Proc. SPIE 9908, 990876 (2016)

22. A. Bechter et al., Proc. SPIE 9909, 99092X (2016)

23. R.A. Probst, T. Steinmetz, T. Wilken, G.K.L. Wong, H. Hundertmark, S.P. Stark, P.St.J. Russell, T.W. Hänsch, R. Holzwarth, Th. Udem, Proc. SPIE 8864, 88641Z (2013)

24. R.A. Probst, Y. Wu, T. Steinmetz, S.P. Stark, T.W. Hänsch, Th. Udem, R. Holzwarth, CLEO 2015, SW4G.7 (2015)

25. J. Reichel, W. Hänsel, Laser physics at the limits (Springer, Berlin, 2002) 